\title{
Inelastic decay rate of quasiparticles in a two-dimensional spin-orbit coupled electron system
}

\author{
I. A. Nechaev, ${ }^{1,2, *}$ P. M. Echenique,,$^{2,3,4}$ and E. V. Chulkov $2,3,4$ \\ ${ }^{1}$ Department of Theoretical Physics, Kostroma State University, 156961 Kostroma, Russia \\ ${ }^{2}$ Donostia International Physics Center (DIPC), P. de Manuel Lardizabal, 4, San Sebastián, 20018 Basque Country, Spain \\ ${ }^{3}$ Departamento de Física de Materiales, Facultad de Ciencias Químicas, UPV/EHU, \\ Apdo. 1072, San Sebastián, 20080 Basque Country, Spain \\ ${ }^{4}$ Centro de Física de Materiales CFM-Materials Physics Center MPC, Centro Mixto CSIC-UPV/EHU, \\ San Sebastián, 20080 Basque Country, Spain \\ (Received 19 September 2009; published 14 May 2010)
}

\begin{abstract}
We present a study of the inelastic decay rate of quasiparticles in a two-dimensional (2D) electron gas with spin-orbit interaction. The study is done within the $G^{0} W^{0}$ approximation. The spin-orbit interaction is taken in the most general form that includes both Rashba and Dresselhaus contributions linear in magnitude of the electron 2D momentum. Spin-orbit interaction effect on the inelastic decay rate is examined at different parameters characterizing the electron gas and the spin-orbit interaction strength in it.
\end{abstract}

DOI: 10.1103/PhysRevB.81.195112

PACS number(s): 71.10.-w, 71.70.Ej

\section{INTRODUCTION}

Nowadays, in condensed-matter physics and semiconductor microelectronics, two-dimensional (2D) electron system is one of the main objects of detailed study. Such a system is formed by, e.g., surface-state electrons or electrons in semiconductor heterostructures. Phenomenon that is observed in such systems and makes them of great interest, especially in context of spintronic applications, is spin-orbit interaction (SOI). This interaction arises from the structure inversion asymmetry of potential confining the electron system in directions perpendicular to the confinement plane (the Rashba spin-orbit interaction ${ }^{1}$ ) and the bulk inversion asymmetry that is present in semiconductor heterostructures based on materials with a zinc-blende structure (the Dresselhaus spinorbit interaction ${ }^{2,3}$ ). The Dresselhaus interaction depends on semiconductor material and growth geometry, whereas the interaction strength of the Rashba SOI can be tuned via an externally applied electric field perpendicular to the confinement plane. ${ }^{4}$ As a result, one can controllably manipulate the spin in devices without recourse to an external magnetic field. 5,6

In order to efficiently exploit the mentioned phenomenon, a theoretical study of dynamics of electrons and holes in the 2D spin-orbit coupled electron systems is needed. The most discussed and studied processes concerning this problem are spin relaxation and spin dephasing. ${ }^{7}$ However, to our knowledge, such crucial quasiparticle property as the lifetime caused by inelastic electron-electron scattering remains still insufficiently studied. To all appearance the first attempt to analyze what effect the SOI has on the quasiparticle lifetime has been made in Ref. 8. In the work cited, a particular case of the 2D electron gas (2DEG) with the Rashba SOI was considered at the limit of $E_{R} \ll E_{F}$, where $E_{F}$ is the Fermi energy and $E_{R}=m^{*} \alpha^{2} / 2$ with $\alpha$ and $m^{*}$ being the interaction strength and the effective electron mass, respectively (unless stated otherwise, atomic units are used throughout, i.e., $e^{2}$ $=\hbar=m=1)$. Within the $G^{0} W^{0}$ approximation, it has been shown that in a small vicinity of $E_{F}$ a modification of the lifetime due to the SOI is insignificant and does not depend on the subband index of the spin-orbit split band. To go beyond the limits of Ref. 8, in Ref. 9 the inelastic lifetime (decay rate) of quasiparticles in the 2DEG with the Rashba SOI has been studied within a wide energy region. For material parameters typical for $\mathrm{In}_{x} \mathrm{Ga}_{1-x}$ As $2 \mathrm{DEGs}$, it has been revealed that modifications induced by the SOI and the dependence on the subband index become noticeable, when the decay channel due to plasmon emission appears. The first joint theoretical and experimental investigation of hole lifetimes in a 2D spin-orbit coupled electron system has been done in Ref. 10. In addition to a demonstration of the weak influence of the SOI on hole lifetimes by the case of the $\mathrm{Au}(111)$ surface state, a hypothetical system, where the SOI can have a profound effect, has been considered.

In this work, we generalize the results on effect of the SOI on the quasiparticle lifetime. Within the $G^{0} W^{0}$ approach with the screened interaction $W^{0}$ evaluated in the random-phase approximation (RPA), we study the inelastic decay rate of quasiparticles in a 2DEG with the Rashba and the Dresselhaus interactions linear in $k$-magnitude of the electron 2D momentum $\mathbf{k}$. In our $G^{0} W^{0}$ calculations, material parameters suitable for InAs quantum wells are taken. We compare the inelastic decay rates calculated at different ratios between the interaction strengths of the mentioned spin-orbit interactions. We show that on the energy scale, for the taken material parameters, the main visible effect induced by the SOI is modifications of the plasmon-emission decay channel via the extension of the Landau damping region. We also consider a hypothetical small-density case, when in the 2D spin-orbit coupled electron system the Fermi level is close to the band energy at $\mathbf{k}=0$. For such a system, we predict strong subband-index dependence and anisotropy of the inelastic decay rate for electrons and appearance of a plasmon decay channel for holes.

\section{APPROXIMATIONS}

We consider a 2DEG described by the Hamiltonian $H$ $=H_{0}+H_{\text {SO }}$ with $H_{0}=k^{2} / 2 m^{*}$ and the spin-orbit contribution 


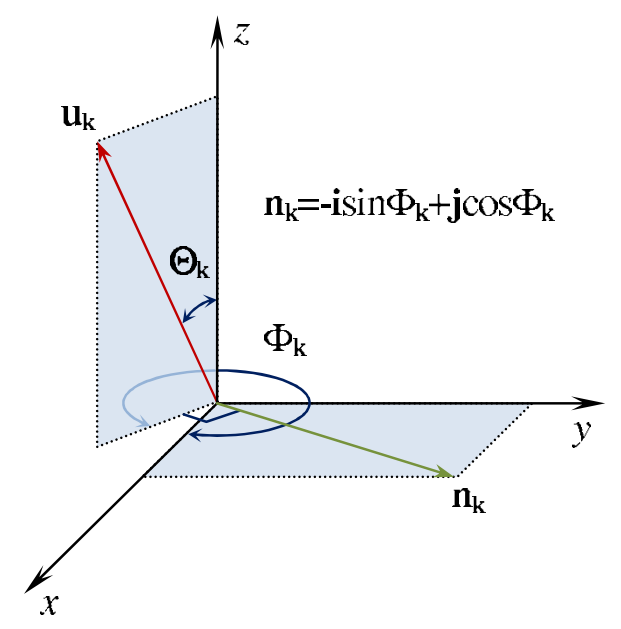

FIG. 1. (Color online) A spatial location of the vectors $\mathbf{u}_{\mathbf{k}}$ and $\mathbf{n}_{\mathbf{k}}$ determining the spin-quantization axis with polar angles $\Theta_{\mathbf{k}}$ and $\Phi_{\mathbf{k}}$ and the rotation axis, respectively.

$$
H_{\mathrm{SO}}=\alpha\left(\sigma_{x} k_{y}-\sigma_{y} k_{x}\right)+\beta\left(\sigma_{x} k_{x}-\sigma_{y} k_{y}\right)
$$

that includes both Rashba and Dresselhaus terms. The latter is written with the assumption that a quantum well grown in the [001] direction is considered. In Eq. (1), $k_{x, y}$ are the electron momenta along the [100] and the [010] cubic axes of the crystal, respectively, $\sigma_{x, y}$ are the Pauli matrices, $m^{*}$ is the effective electron mass, $\alpha$ and $\beta$ are the interaction strengths for the Rashba and the Dresselhaus spin-orbit interactions. To bring the Hamiltonian to a diagonal form, we perform the rotation in spin space generated by $U_{\mathbf{k}}=\exp \left[i\left(\boldsymbol{\sigma} \cdot \mathbf{n}_{\mathbf{k}}\right) \Theta_{\mathbf{k}} / 2\right]$ dependent on the momentum $\mathbf{k}$. The rotation is performed with the angle $\Theta_{\mathbf{k}}$ around the axis determined by $\mathbf{n}_{\mathbf{k}}$. A positional relationship of the axis $\mathbf{n}_{\mathbf{k}}$ and the spin-quantization axis $\mathbf{u}_{\mathbf{k}}$ is shown in Fig. 1. We suppose that we deal with the in-plane spin polarization, i.e., $\Theta_{\mathbf{k}}=\pi / 2$. In the new, unitary transformed, spin basis the spin-orbit contribution has the form ${ }^{11}$

$$
H_{\mathrm{SO}}^{\prime}=U_{\mathbf{k}}^{\dagger} H_{\mathrm{SO}} U_{\mathbf{k}}=-k\left[\alpha \sin \left(\varphi_{\mathbf{k}}-\Phi_{\mathbf{k}}\right)+\beta \cos \left(\varphi_{\mathbf{k}}+\Phi_{\mathbf{k}}\right)\right] \sigma_{z},
$$

where the angle $\Phi_{\mathbf{k}}$ is related to the polar angle $\varphi_{\mathbf{k}}$ of the momentum $\mathbf{k}$ as

$$
\tan \Phi_{\mathbf{k}}=-\frac{\alpha \cos \varphi_{\mathbf{k}}+\beta \sin \varphi_{\mathbf{k}}}{\alpha \sin \varphi_{\mathbf{k}}+\beta \cos \varphi_{\mathbf{k}}} .
$$

Due to the diagonal form of $H_{\mathrm{SO}}^{\prime}$, the energy bands are simply given by ${ }^{12}$

$$
E_{\mathbf{k} s}=\frac{\mathbf{k}^{2}}{2 m^{*}}+s k\left[\alpha \sin \left(\varphi_{\mathbf{k}}-\Phi_{\mathbf{k}}\right)+\beta \cos \left(\varphi_{\mathbf{k}}+\Phi_{\mathbf{k}}\right)\right]
$$

and correspond to the wave functions $\psi_{\mathbf{k} s}^{\prime}(\mathbf{r})=e^{i \mathbf{k r}}|-s\rangle$ with the subband index $s= \pm(\downarrow, \uparrow)$, where $\downarrow, \uparrow$ are the spin components in the new spin basis. This means that for the initial, untransformed, Hamiltonian we have the following eigenstates $\psi_{\mathbf{k} s}(\mathbf{r})=U_{\mathbf{k}} \psi_{\mathbf{k} s}^{\prime}(\mathbf{r})$. The spin orientation in $\mathbf{k}$ space reads as (see Fig. 2)
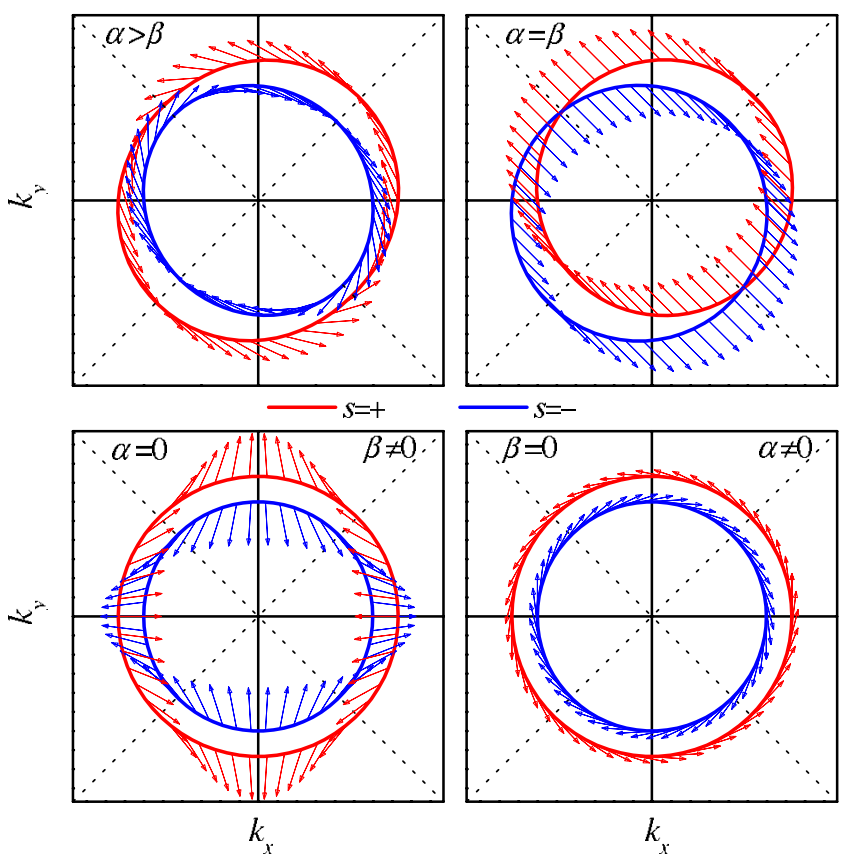

FIG. 2. (Color online) Fermi contours and spin orientations in the momentum plane for different values of interaction strengths of the Rashba and the Dresselhaus spin-orbit interactions.

$$
\left\langle\psi_{\mathbf{k} s}|\sigma| \psi_{\mathbf{k} s}\right\rangle=s\left(\begin{array}{c}
\cos \Phi_{\mathbf{k}} \\
\sin \Phi_{\mathbf{k}} \\
0
\end{array}\right) .
$$

Note that the case with $\alpha \neq 0$ and $\beta=0$ (pure Rashba) is characterized by the angle $\Phi_{\mathbf{k}}=\varphi_{\mathbf{k}}-\pi / 2$, whereas in the situation with $\beta \neq 0$ and $\alpha=0$ (pure Dresselhaus) one has $\Phi_{\mathbf{k}}$ $=2 \pi-\varphi_{\mathbf{k}}$. In the special case of $\alpha=\beta$, the angle $\Phi_{\mathbf{k}}=-\pi / 4$ does not depend on $\varphi_{\mathbf{k}}$.

The inelastic decay rate [inverse lifetime $\tau_{s}^{-1}(\mathbf{k})$ caused by inelastic electron-electron scattering] is determined by the imaginary part of the matrix elements of the quasiparticle self-energy $\left\langle\Sigma_{s}(\mathbf{k}, \omega)\right\rangle=\left\langle\psi_{\mathbf{k} s}\left(\mathbf{r}_{1}\right)\left|\Sigma\left(\mathbf{r}_{1}, \mathbf{r}_{2} ; \omega\right)\right| \psi_{\mathbf{k} s}\left(\mathbf{r}_{2}\right)\right\rangle_{\mathbf{r}_{1} \mathbf{r}_{2}}$ at the energy $\omega=E_{\mathbf{k} s}$ as $\Gamma_{s}(\mathbf{k})=2\left|\operatorname{Im}\left\langle\Sigma_{s}\left(\mathbf{k}, E_{\mathbf{k} s}\right)\right\rangle\right|$. At the Hartree-Fock (HF) mean-field level, these elements are totally real and have the form

$$
\left\langle\Sigma_{s}^{\mathrm{HF}}(\mathbf{k})\right\rangle=-\sum_{s^{\prime}} \int \frac{d \mathbf{q}}{(2 \pi)^{2}} F_{\mathbf{k}, \mathbf{q}}^{s s^{\prime}} f_{\mathbf{q} s^{\prime}} v_{c}(\mathbf{k}-\mathbf{q}),
$$

where $v_{c}(\mathbf{k})=2 \pi /\left(|\mathbf{k}| \varepsilon_{0}\right)$ is the bare Coulomb interaction with $\varepsilon_{0}$ being the static dielectric constant. The factors $F_{\mathbf{k}, \mathbf{p}}^{s s^{\prime}}$ $=\left[1+s s^{\prime} \mathbf{u}_{\mathbf{k}} \cdot \mathbf{u}_{\mathbf{p}}\right] / 2$ come from $\left|\left\langle s^{\prime}\left|U_{\mathbf{p}}^{\dagger} U_{\mathbf{k}}\right| s\right\rangle\right|^{2}$ and $f_{\mathbf{k} s}$ is the Fermi factor. Such a form in Eq. (6) is similar to the exchange contribution to the single-particle energies considered in Refs. 13-15 in the pure Rashba case. However, in order to examine quasiparticle lifetimes, one has to go beyond the HF approximation. The simplest variant is the $G^{0} W^{0}$ approximation (for details about the approximation, we refer the reader to Refs. 9 and 16). Within such an approximation, we arrive at the following expression for the imaginary part of the mentioned matrix elements: 


$$
\begin{aligned}
\operatorname{Im}\left\langle\Sigma_{s}(\mathbf{k}, \omega)\right\rangle= & -\sum_{s^{\prime}} \int \frac{d \mathbf{q}}{(2 \pi)^{2}} F_{\mathbf{k}, \mathbf{q}}^{s \mathbf{q}^{\prime}} f_{\mathbf{q} s^{\prime}} \\
& \times \operatorname{Im} W^{0}\left(\mathbf{k}-\mathbf{q}, \omega-E_{\mathbf{q} s^{\prime}}\right) \theta\left(E_{\mathbf{q} s^{\prime}}-\omega\right),
\end{aligned}
$$

when $\omega<E_{F}$ and

$$
\begin{aligned}
\operatorname{Im}\left\langle\Sigma_{s}(\mathbf{k}, \omega)\right\rangle= & \sum_{s^{\prime}} \int \frac{d \mathbf{q}}{(2 \pi)^{2}} F_{\mathbf{k}, \mathbf{q}}^{s s^{\prime}}\left[1-f_{\mathbf{q} s^{\prime}}\right] \\
& \times \operatorname{Im} W^{0}\left(\mathbf{k}-\mathbf{q}, \omega-E_{\mathbf{q} s^{\prime}}\right) \theta\left(\omega-E_{\mathbf{q} s^{\prime}}\right),
\end{aligned}
$$

when $\omega>E_{F}$. In these equations, $\theta(x)$ is the step function and the screened interaction

$$
W^{0}(\mathbf{q}, \omega)=v_{c}(\mathbf{q})\left[1-P^{0}(\mathbf{q}, \omega) v_{c}(\mathbf{q})\right]^{-1}
$$

is defined by the RPA irreducible polarizability (see also Refs. 17 and 18, where the retarded part of $P^{0}$ was examined)

$$
\begin{aligned}
P^{0}(\mathbf{q}, \omega)= & \sum_{s s^{\prime}} \int \frac{d \mathbf{k}}{(2 \pi)^{2}} F_{\mathbf{k}, \mathbf{k}+\mathbf{q}}^{s s^{\prime}}\left\{\frac{\left(1-f_{\mathbf{k}+\mathbf{q} s}\right) f_{\mathbf{k} s^{\prime}}}{\omega+E_{\mathbf{k} s^{\prime}}-E_{\mathbf{k}+\mathbf{q} s}+i \eta}\right. \\
& \left.-\frac{f_{\mathbf{k}+\mathbf{q} s}\left(1-f_{\mathbf{k} s^{\prime}}\right)}{\omega+E_{\mathbf{k} s^{\prime}}-E_{\mathbf{k}+\mathbf{q} s}-i \eta}\right\} .
\end{aligned}
$$

\section{RESULTS AND DISCUSSION}

Using the example of an InAs quantum well, we take the effective mass $m^{*}=0.023$ (see, e.g., Ref. 19) and the static dielectric constant $\varepsilon_{0}=14.55$ (see, e.g., Ref. 20). The interaction strength of the Dresselhaus SOI is chosen to be $\beta=1.6$ $\times 10^{-11} \mathrm{eV} \mathrm{m}$ to simulate a quite narrow quantum well. ${ }^{19} \mathrm{At}$ the ratio $\alpha / \beta=2.4$ (see, e.g., Ref. 21), we have the Rashba interaction strength $\alpha=3.8 \times 10^{-11} \mathrm{eV} \mathrm{m}$. The electron density is put at $n_{2 \mathrm{D}}=2.55 \times 10^{11} \mathrm{~cm}^{-2}$ that corresponds to the Fermi energy $E_{F} \approx 26 \mathrm{meV}$.

Figure 3 shows our results on the inelastic decay rate $\Gamma_{s}(\mathbf{k})$ obtained with the material parameters listed above. Two main points caused by the spin-orbit splitting of the band make the considered 2DEG different from that without the SOI. These are an angle-dependent relative shift of $\Gamma_{+}$ and $\Gamma_{-}$on the momentum scale and some smoothing of sharp forms of the peak caused by opening of the plasmon decay channel. The former reflects the fact that subbands of the split band reach the same energy at different momenta, while the latter originates from the extension of the Landau damping region (the region where plasmons decay into singleparticle excitations ${ }^{22}$ ) due to appearance of intersubband transitions (for a detailed discussion of the screening properties of the 2DEG with the SOI we refer the reader to Refs. 17 and 18). This extension varying with the polar angle $\varphi_{\mathbf{k}}$ leads to a nonzero plasmon linewidth, when the plasmon spectrum enters into the SOI-induced damping region.

In order to show what effect the SOI has on the inelastic decay rate for different subbands, in the inset of Fig. 3, by setting up a correspondence between $\Gamma_{s}(\mathbf{k})$ and $E_{\mathbf{k} s}$ via the momentum $\mathbf{k}$, we plot the decay rate as a function of energy. On first glance, it may seem that we have an ordinary energy

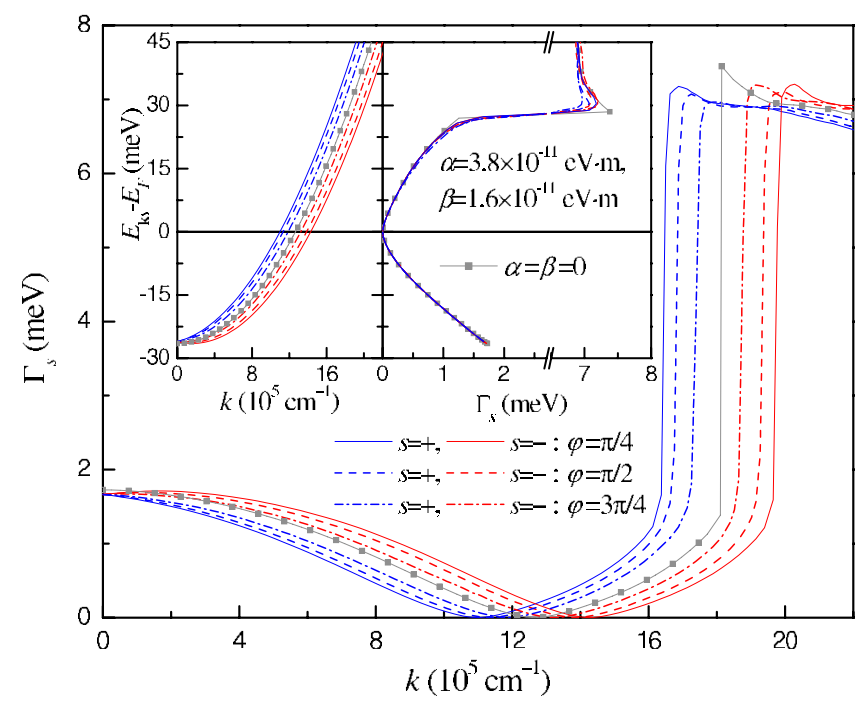

FIG. 3. (Color online) Inelastic decay rate $\Gamma_{s}$ as a function of $k$ at several values of the polar angle $\varphi_{\mathbf{k}}$ in the case of the ratio $\alpha / \beta=2.4$. Inset: the corresponding energy bands $E_{\mathbf{k} s}$ (at the left) and the same $\Gamma_{s}$ as a function of $E_{\mathbf{k} s}$ measured from the Fermi energy (at the right). Also, for reference, the case of the 2DEG without the SOI $(\alpha=\beta=0)$ is presented.

dependence of the decay rate as in a 2DEG without the SOI: the quadratic behavior with the logarithmic enhancement in the vicinity of the Fermi energy with $\Gamma_{s}=0$ at $E_{F}$ and the jump above the Fermi energy, which is caused by opening the plasmon decay channel for excited electrons. ${ }^{23}$ However, on examining the energy dependence of $\Gamma_{s}$ in detail, we can say that due to the finite plasmon linewidth the plasmondecay channel manifests itself at lower energies, when it occurs in a 2DEG without the SOI. The same reason leads to reduction in the jump. Also, we can reveal distinctions between $\Gamma_{+}$and $\Gamma_{-}$, which become noticeable, when the plasmon-emission decay channel appears, and increase upon moving from $\varphi_{\mathbf{k}}=\pi / 4$ to $3 \pi / 4$. An analysis of the inelastic mean free path (IMFP) $\lambda_{s}(\mathbf{k})=\left|\nabla_{\mathbf{k}} E_{\mathbf{k} s}\right| / \Gamma_{s}(\mathbf{k})$ as a function of energy has shown that, as a consequence of the distinctions between $\Gamma_{+}$and $\Gamma_{-}$, the IMFP of electrons can vary with the subband index. For example, at $\varphi_{\mathbf{k}}=3 \pi / 4$ for electrons this variation can reach, e.g., $\sim 8 \%$.

The obtained results can be understood by inspecting constant-energy contours shown in Fig. 2 with mental drawing of possible transitions selected by the factors $F_{\mathbf{k}, \mathbf{q}}^{s^{\prime}}$ of Eqs. (7) and (8) at a given $\varphi_{\mathbf{k}}$ (see also Ref. 10). Actually, for each subband $(s= \pm)$ one has a set of intrasubband and intersubband transition momenta as arguments of $\operatorname{Im} W^{0}$. For the chosen material parameters and for $\varphi_{\mathrm{k}}=\pi / 4$, these momenta do not vary considerably with the subband index $s$. For $\varphi_{\mathbf{k}}$ $=3 \pi / 4$ differences in both intrasubband and intersubband transitions for $s=+$ and $s=-$ become already sensible for values of $\mathrm{Im} W^{0}$, especially in the vicinity of plasmon peaks of the latter.

Now, remaining $m^{*}, \varepsilon_{0}, n_{2 \mathrm{D}}$, and $E_{F}$ unchanged, we consider the case of $\alpha / \beta=1\left[\alpha=\beta=3.0 \times 10^{-11} \mathrm{eV} \mathrm{m}\right]$ and the pure Dresselhaus (Rashba) case $\left[\beta(\alpha)=4.2 \times 10^{-11} \mathrm{eV} \mathrm{m}\right.$ and $\alpha(\beta)=0]$. The case of equal interaction strengths, when 


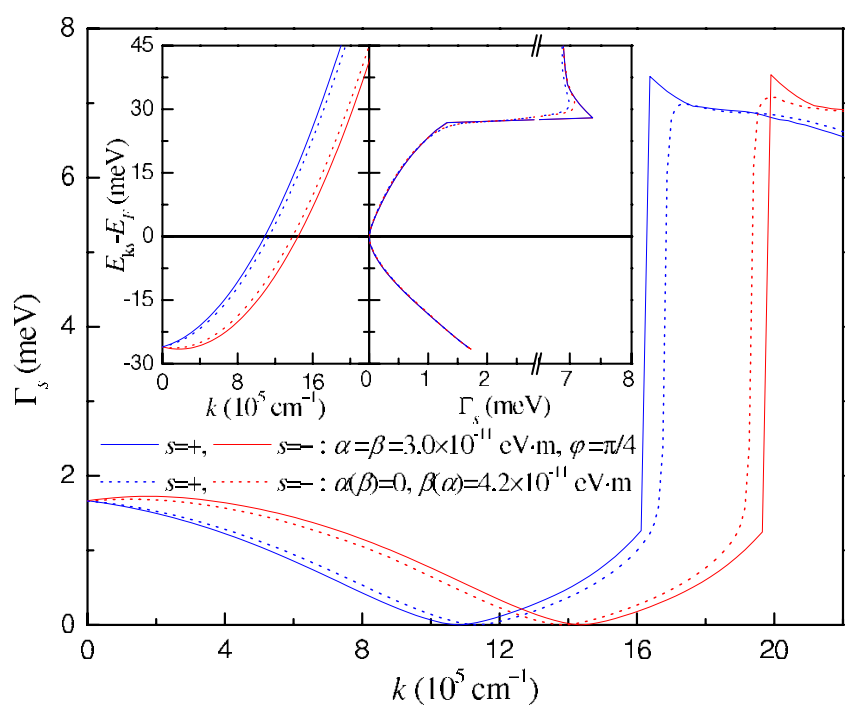

FIG. 4. (Color online) Same as in Fig. 3, but for the ratio $\alpha / \beta$ $=1$. Also, the pure Rashba and the pure Dresselhaus cases are presented.

the Rashba and the Dresselhaus interactions can cancel each other, is distinguished by various significant effects reported in the literature (see, e.g., Refs. 24-27). In this case, one has the $2 \mathrm{D}$ electron system with two uncoupled spin components (see Fig. 2), each of which demonstrates the properties peculiar to a 2 DEG without the SOI. ${ }^{18}$ Our results ${ }^{28}$ on the inelastic decay rate at $\alpha / \beta=1$ are shown in Fig. 4. The sharp edges of the plasmon contribution are evidence of the fact that there is no modification of the Landau damping region induced by the SOI. As is seen from the inset of the figure, due to the shifting property $E_{\mathbf{k}+}=E_{\mathbf{Q}+\mathbf{k}-}$, where $Q_{x}=Q_{y}$ $=\sqrt{8} m^{*} \alpha, \Gamma_{+}$and $\Gamma_{-}$curves coincide and have the form of that in a 2 DEG without the SOI.

In the pure Rashba or the pure Dresselhaus cases (see Fig. 4 ), the resulting $\Gamma_{s}$ does not tell the difference between spin orientations in the momentum plane, which correspond to the Rashba or the Dresselhaus SOI (see Fig. 2). As well as before, we have the relative shift (but angle independent) on the momentum scale and main modifications induced by the SOI in the energy region, where a quasiparticle can decay into plasmons.

All the considered cases meet the condition of $E_{R n D}$ $\ll E_{F}$, where $E_{R n D}=m^{*}(|\alpha|+|\beta|)^{2} / 2$ is the measure of influence of the SOI on the band structure. However, as is partly discussed in Ref. 10, in two-dimensional electron systems with much greater $E_{R n D}$ as compared to $E_{F}$ the inelastic decay rate can substantially differ from that in the 2DEG without the SOI. A striking example of such a system is that formed by surface-state electrons in ordered surface alloys, ${ }^{29}$ which are very promising materials for spintronics applications. In order to predict how the inelastic decay rate can behave in a system, where $E_{R n D} \sim E_{F}$, we consider the hypothetical case with the unchanged $m^{*}=0.023, \varepsilon_{0}=14.55$, and $\beta=1.6 \times 10^{-11} \mathrm{eVm}$, but with $\alpha=5.9 \times 10^{-11} \mathrm{eV} \mathrm{m}(\alpha / \beta$ $=3.7)$ and $n_{2 \mathrm{D}}=2.04 \times 10^{10} \mathrm{~cm}^{-2}$, which give $E_{F}=1.0 \mathrm{meV}$ and $E_{R n D}=0.85 \mathrm{meV}$. The obtained results are presented in Fig. 5.

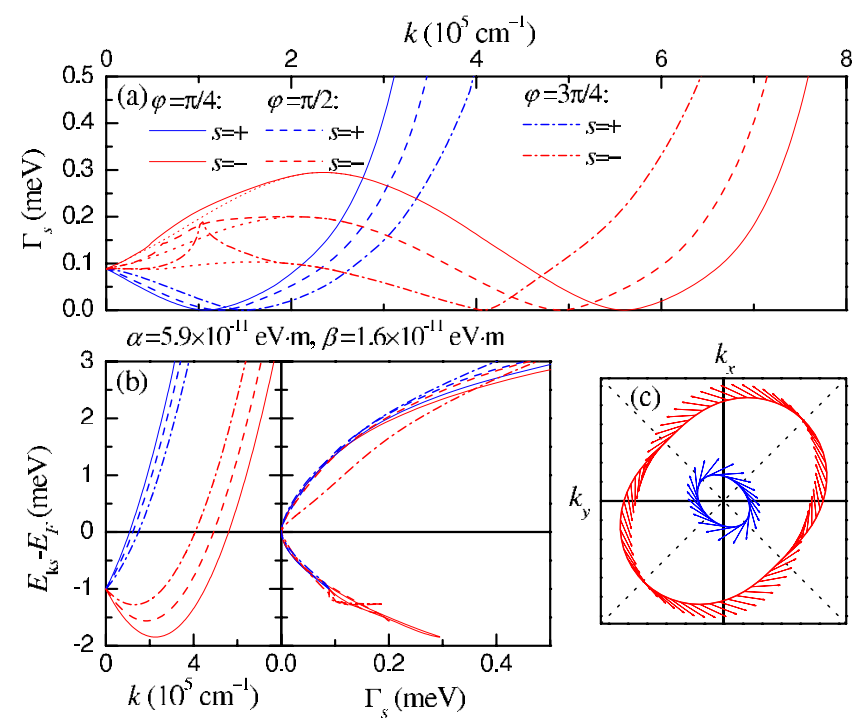

FIG. 5. (Color online) (a) Inelastic decay rate $\Gamma_{s}$ as a function of $k$ at several values of the polar angle $\varphi_{\mathbf{k}}$ in the small electron density case at $\alpha / \beta=3.7$. Dotted lines represent $\Gamma_{s}$ without the plasmon contribution (see the text). (b) The corresponding energy bands $E_{\mathbf{k} s}$ (at the left) and the same $\Gamma_{s}$ as a function of $E_{\mathbf{k} s}$ measured from the Fermi energy (at the right). (c) Spin orientations in the momentum plane for the considered electron gas.

The main feature we would like to note first is that for holes the decay rate $\Gamma_{\text {- }}$ as a function of $k$ has an "outgrowth" at $k<k_{\text {min }}$, where $k_{\text {min }} \equiv m^{*}\left[\alpha^{2}+\beta^{2}+2 \alpha \beta \sin \left(2 \varphi_{\mathbf{k}}\right)\right]^{1 / 2}$ - the momentum, at which $E_{\mathbf{k}-}$ has a minimum. A close analysis of the imaginary part of the screened interaction $W^{0}$ and the region of integration in Eq. (7) has shown that the outgrowth is caused by opening of the plasmon decay channel for transitions between the $s=-$ and $s^{\prime}=+$ subbands. It is important that in a 2 DEG without the SOI such a channel is impossible for holes. ${ }^{23}$

As is evident from the figure, in this case on the energy scale we have a strong anisotropy of the inelastic decay rate. Also the presented curves clearly demonstrate that the latter depends strongly on the subband index $s$ of the spin-orbit split band. Keeping in mind that the index $s$ distinguishes spin components, we can say that the subband-index dependence reflects a spin asymmetry of the inelastic decay rate $\Gamma_{-} / \Gamma_{+}$for a given direction. The asymmetry shows its worth most brightly in the $\varphi_{\mathbf{k}}=3 \pi / 4$ direction. In fact, in that very direction there are significant distinctions in $\Gamma_{-}$and $\Gamma_{+}$as functions of the exciting energy and, as a consequence, in the corresponding IMFP for electrons. For instance, the ratio $\Gamma_{-} / \Gamma_{+}$is about 3 at $\sim 0.5 \mathrm{meV}$ and about 2 at $\sim 1.0 \mathrm{meV}$. At further increasing of energy, the ratio continues to decrease.

Note that the IMFP spin asymmetry makes a basis of the spin filter effect observed in hot-electron transport through a ferromagnetic (see, e.g., Refs. 7 and 30). In the considered case of the quantum well, the spin asymmetry is not such big as in ferromagnetics (see, e.g., Ref. 31), but, as distinct from the latter, values of the IMFP spin symmetry depend strongly on direction and can be tuned by external electric field. 


\section{CONCLUSIONS}

In conclusion, we have presented a study of the inelastic decay rate of quasiparticles in a two-dimensional electron gas with the $k$-linear spin-orbit interaction that includes both Rashba (interaction strength $\alpha$ ) and Dresselhaus (interaction strength $\beta$ ) contributions. In this study, the electron gas is characterized by material parameters suitable for [001]grown InAs quantum wells. We have considered the cases of $\alpha>\beta>0, \alpha=\beta, \alpha=0(\beta>0)$, and $\beta=0(\alpha>0)$. The cases meet the condition of $E_{R n D} \ll E_{F}$, where $E_{R n D}=m^{*}(|\alpha|$ $+|\beta|)^{2} / 2$ is the measure of influence of the spin-orbit interaction on the band structure.

As compared to a two-dimensional electron gas without the spin-orbit interaction, we have revealed a relative shift of the inelastic decay rates for different subbands of the spinorbit split band on the momentum scale. Also, except for the case of equal interaction strengths, we have found some smoothing of sharp forms of the peak concerned with opening of the plasmon decay channel for electrons. We have shown that, on the energy scale, in this very region distinctions between the decay rates for different subbands become noticeable. These distinctions depend on the polar angle $\varphi_{\mathbf{k}}$ and cause the inelastic mean free path to be angle and sub- band dependent. As to the case of $\alpha=\beta$, due to the shifting property, the decay rate as a function of energy has the form of that in a two-dimensional electron gas without the spinorbit interaction.

In order to predict how the inelastic decay rate can behave in a system, where $E_{R n D} \sim E_{F}$, we have considered the hypothetical case of small electron density. We have revealed that in such a system the decay rate demonstrates strong anisotropy and subband dependence within all the considered interval of momenta and exciting energies. Since the subband dependence can be interpreted as a spin asymmetry of the decay rate in a given direction of $\mathbf{k}$, one can expect the spin-filter effect driven by externally applied electric field. Also, we have found that in the system with $E_{R n D} \sim E_{F}$ holes can decay into plasmons, what is impossible in a twodimensional electron gas without the spin-orbit interaction.

\section{ACKNOWLEDGMENTS}

We acknowledge partial support from the University of the Basque Country (Grant No. GIC07IT36607) and the Spanish Ministerio de Ciencia y Tecnología (Grant No. FIS2007-66711-C02-01). Calculations were partly performed on SKIF-Cyberia supercomputer of Tomsk State University.
*Also at REC "Physics and Chemistry of High-Energy Systems," Tomsk State University, 634050 Tomsk, Russia.

${ }^{1}$ E. I. Rashba, Sov. Phys. Solid State 2, 1109 (1960); Y. A. Bychkov and E. I. Rashba, JETP Lett. 39, 78 (1984); J. Phys. C 17, 6039 (1984).

${ }^{2}$ G. Dresselhaus, Phys. Rev. 100, 580 (1955).

${ }^{3}$ M. I. Dyakonov and V. Y. Kacharovskii, Sov. Phys. Semicond. 20, 110 (1986).

${ }^{4}$ M. Studer, G. Salis, K. Ensslin, D. C. Driscoll, and A. C. Gossard, Phys. Rev. Lett. 103, 027201 (2009).

${ }^{5}$ S. Datta and B. Das, Appl. Phys. Lett. 56, 665 (1990).

${ }^{6}$ J. Nitta, T. Akazaki, H. Takayanagi, and T. Enoki, Phys. Rev. Lett. 78, 1335 (1997).

${ }^{7}$ I. Žutić, J. Fabian, and S. Das Sarma, Rev. Mod. Phys. 76, 323 (2004).

${ }^{8}$ D. S. Saraga and D. Loss, Phys. Rev. B 72, 195319 (2005).

${ }^{9}$ I. A. Nechaev and E. V. Chulkov, Phys. Solid State 51, 1772 (2009).

${ }^{10}$ I. A. Nechaev, M. F. Jensen, E. D. L. Rienks, V. M. Silkin, P. M. Echenique, E. V. Chulkov, and Ph. Hofmann, Phys. Rev. B 80, 113402 (2009).

${ }^{11}$ In the case of $\alpha=\beta$ the Hamiltonian $H$ in the new spin basis and the unitary transformation $U_{\mathbf{k}}$ become exactly the same as $\mathcal{H}_{[\operatorname{Re~D}]}$ and $U$ of Ref. 24, respectively.

${ }^{12}$ Note that Eq. (4) can be cast into the form that is frequently used in the literature. Actually, from $\left(E_{\mathbf{k} s}-\mathbf{k}^{2} / 2 m^{*}\right)^{2}=\left(k\left[\alpha \sin \left(\varphi_{\mathbf{k}}\right.\right.\right.$ $\left.\left.\left.-\Phi_{\mathbf{k}}\right)+\beta \cos \left(\varphi_{\mathbf{k}}+\Phi_{\mathbf{k}}\right)\right]\right)^{2}$ with the help of Eq. (3) we arrive at $\left(E_{\mathbf{k} s}-\mathbf{k}^{2} / 2 m^{*}\right)^{2}=k^{2}\left[\alpha^{2}+\beta^{2}+2 \alpha \beta \sin \left(2 \varphi_{\mathbf{k}}\right)\right]$. The latter can be solved as $E_{\mathbf{k} s}=\mathbf{k}^{2} / 2 m^{*}+s k\left[\alpha^{2}+\beta^{2}+2 \alpha \beta \sin \left(2 \varphi_{\mathbf{k}}\right)\right]^{1 / 2}$. However, in such an expression the subband index $s= \pm$ distinguishes the inner and outer branch and, e.g., in the case of $\alpha=\beta$ does not correspond to spin components.

${ }^{13}$ G.-H. Chen and M. E. Raikh, Phys. Rev. B 60, 4826 (1999).

${ }^{14}$ S. Chesi and G. F. Giuliani, Phys. Rev. B 75, 155305 (2007).

${ }^{15}$ L. O. Juri and P. I. Tamborenea, Phys. Rev. B 77, 233310 (2008).

${ }^{16}$ I. A. Nechaev, I. Yu. Sklyadneva, V. M. Silkin, P. M. Echenique, and E. V. Chulkov, Phys. Rev. B 78, 085113 (2008).

${ }^{17}$ M. Pletyukhov and V. Gritsev, Phys. Rev. B 74, 045307 (2006).

${ }^{18}$ S. M. Badalyan, A. Matos-Abiague, G. Vignale, and J. Fabian, Phys. Rev. B 79, 205305 (2009).

${ }^{19}$ W. Knap, C. Skierbiszewski, A. Zduniak, E. Litwin-Staszewska, D. Bertho, F. Kobbi, J. L. Robert, G. E. Pikus, F. G. Pikus, S. V. Iordanskii, V. Mosser, K. Zekentes, and Yu. B. Lyanda-Geller, Phys. Rev. B 53, 3912 (1996).

${ }^{20}$ O. G. Lorimor and W. G. Spitzer, J. Appl. Phys. 36, 1841 (1965).

${ }^{21}$ S. Giglberger, L. E. Golub, V. V. Bel'kov, S. N. Danilov, D. Schuh, C. Gerl, F. Rohlfing, J. Stahl, W. Wegscheider, D. Weiss, W. Prettl, and S. D. Ganichev, Phys. Rev. B 75, 035327 (2007).

${ }^{22} \mathrm{H}$. Bruus and K. Flensberg, Many-Body Quantum Theory in Condensed Matter Physics: An Introduction (Oxford University Press, Oxford, 2004).

${ }^{23}$ G. F. Giuliani and G. Vignale, Quantum Theory of the Electron Liquid (Cambridge University Press, Cambridge, 2005).

${ }^{24}$ B. A. Bernevig, J. Orenstein, and S.-C. Zhang, Phys. Rev. Lett. 97, 236601 (2006).

${ }^{25}$ N. S. Averkiev and L. E. Golub, Phys. Rev. B 60, 15582 (1999).

${ }^{26}$ J. Schliemann, J. C. Egues, and D. Loss, Phys. Rev. Lett. 90, 146801 (2003). 
${ }^{27}$ J. D. Koralek, C. P. Weber, J. Orenstein, B. A. Bernevig, S.-C. Zhang, S. Mack, and D. D. Awschalom, Nature (London) 458, 610 (2009).

${ }^{28}$ We have chosen the direction $\varphi_{\mathbf{k}}=\pi / 4$, in which we can reproduce the momentum dependence of the inelastic decay rate peculiar for a 2DEG without the SOI. For each spin component, such a dependence becomes isotropic if one shifts the origin in the momentum plane on the vector $s \mathbf{Q} / 2$.

${ }^{29}$ C. R. Ast, J. Henk, A. Ernst, L. Moreschini, M. C. Falub, D.
Pacilé, P. Bruno, K. Kern, and M. Grioni, Phys. Rev. Lett. 98, 186807 (2007); C. R. Ast, D. Pacilé, L. Moreschini, M. C. Falub, M. Papagno, K. Kern, M. Grioni, J. Henk, A. Ernst, S. Ostanin, and P. Bruno, Phys. Rev. B 77, 081407(R) (2008); H. Mirhosseini, J. Henk, A. Ernst, S. Ostanin, C.-T. Chiang, P. Yu, A. Winkelmann, and J. Kirschner, ibid. 79, 245428 (2009). ${ }^{30}$ V. P. Zhukov and E. V. Chulkov, Phys. Usp. 52, 105 (2009).

${ }^{31}$ I. A. Nechaev and E. V. Chulkov, Phys. Solid State 51, 754 (2009). 